\title{
Zein nanoparticles for oral folic acid delivery
}

\section{Authors}

Rebeca Peñalva a, Irene Esparza a, Carlos J. González-Navarro ${ }^{b}$, Gemma Quincoces ${ }^{c}$, Ivan Peñuelas ${ }^{c}$, Juan M. Irache ${ }^{a}$

\section{Affiliation}

a Department of Pharmacy and Pharmaceutical Technology, University of Navarra, 31008 - Pamplona, Spain.

b Centre for Nutrition Research, University of Navarra, 31080 - Pamplona, Spain.

${ }^{c}$ Radiopharmacy Unit, University Clinic of Navarra. Av. Pío XII, 36. 31008 Pamplona, Spain

\section{Corresponding author:}

Prof. Juan M. Irache

Dep. Pharmacy and Pharmaceutical Technology

University of Navarra

C/ Irunlarrea, 1

31080 - Pamplona

Spain

Phone: +34948425600

Fax: +34948425619

E-mail: jmirache@unav.es

Short title: Zein nanoparticles and folic acid 


\section{Abstract}

32 The aim of this work was to prepare and evaluate the capability of zein 33 nanoparticles for oral drug delivery. More particularly, in this work, the ability of 34 these nanoparticles to improve the oral bioavailability of folic acid is reported. 35 The nanoparticles were prepared by a desolvation process, followed by 36 purification via ultrafiltration and drying in a spray-drier apparatus. The resulting 37 nanoparticles displayed a mean size close to $200 \mathrm{~nm}$ with negative zeta 38 potential and a payload of $54 \mu \mathrm{g}$ folic acid per mg nanoparticle. From the in vitro 39 release studies, it was observed that folic acid was only released from 40 nanoparticles in simulated intestinal conditions. In vivo biodistribution studies, 41 with radiolabelled or fluorescently marked nanoparticles, revealed that 42 nanoparticles remained within the gut and were capable of interacting with the 43 protective mucus layer of the jejunum. For the pharmacokinetic study, folic acid 44 was orally administered to rats as a single dose of $1 \mathrm{mg} / \mathrm{kg}$.

45 The relatively oral bioavailability of folic acid, when encapsulated in zein 46 nanoparticles, was around $70 \%$ : two-times higher than the value obtained with 47 an aqueous solution of the vitamin. This fact might be explained by the 48 mucoadhesive properties of these nanoparticles.

49

Key words: zein; nanoparticles; folic acid; bioavailability; biodistribution; mucoadhesion 


\section{Introduction}

Zein, the major storage protein of maize, is located in the "zein-bodies", of approximately $1 \mu \mathrm{m}$, that are distributed uniformly throughout the cytoplasm of the corn endosperm cells between starch granules of 5-35 $\mu \mathrm{m}$ [1]. From a physicochemical point of view, the key characteristic of zein is its insolubility in water except at extreme $\mathrm{pH}$ conditions (e.g., $\mathrm{pH} 11$ or above) or in presence of high concentrations of urea, alcohol or anionic detergents [2]. This characteristic is directly related with its composition in amino acids. Thus, zein is particularly rich in glutamic acid (21-26\%) and non-polar amino acids such as leucine $(20 \%)$, proline $(10 \%)$ and alanine $(10 \%)$, but it is deficient in basic and acidic amino acids [3].

Actually, zein is not a single protein but a mixture of four main fractions ( $\alpha-, \beta-$, $\gamma-$, and $\delta$-zein) that differentiated in their solubility and sequence $[1,4]$. Alphazein is the most abundant (around $80 \%$ of total zein) and includes two prolamin groups with apparent molecular weights of 24 and $27 \mathrm{kDa}$. Beta-zein consists of a methionine-rich polypeptide of $17 \mathrm{kDa}$ and constitutes up to $10 \%$ of the total zein; whereas $\mathrm{y}$-zein is also composed of two peptides of 27 and $18 \mathrm{kDa}$. Finally, $\delta$-zein is a minor fraction and has a molecular weight of about $10 \mathrm{kDa}$ $[1,4,5]$.

Because of its hydrophobic character and deficiency in essential amino acids (e.g. lysine and tryptophan), the use of this corn protein in human food products is limited. However, zein has been proposed as material for the manufacture of a wide variety of products, including textile fibers for clothes [6], biodegradable films and plastics used for packaging [7], coatings for food and pharmaceutical dosage forms $[8,9]$ and scaffolds for tissue engineering [10].

In the last years, microparticles and nanoparticles from zein have also been studied as carriers of non-polar compounds including vitamin D3 [11], curcumin [12] or thymol [13]. Such devices were capable of protecting the loaded compounds from stomach harsh conditions and providing a mechanism for their controlled release $[14,15]$.

Folic acid (pteroyl-L-glutamic acid, vitamin B9) is a water soluble vitamin that is essential during periods of rapid cell division and growth. It is implicated in cell replication and has an important role in the one-carbon metabolic pathway, essential for cardiovascular and neurological functions [16]. During periods of inadequate folate intake or malabsorption, biochemical changes due to this lack of folic acid/folate may result in deleterious consequences, including increased risk for certain types of chronic diseases [17] and developmental disorders (e.g., neural tube defects) [18]. In this way, previous studies have shown that folate deficiency is associated with higher incidence of mental symptoms in general population and poor cognitive performance that may increase the risk of dementia in old age $[18,19]$. Particularly in major depression, low folic acid levels are frequently described in clinical studies [20]. Corroborating these findings, a variety of controlled and open-label studies have shown that the efficacy of antidepressants is influenced by folate status and may be enhanced by folic acid supplementation [21]. On the other hand, low folate intake or low plasma folate concentration has also been associated with increased cardiovascular and cerebrovascular risks [22]. All of these effects would be related with high plasma levels of homocysteine, a cytotoxic sulfur-containing 
amino acid that can induce DNA strand breakage, oxidative stress and apoptosis [22].

Interestingly, folic acid supplementation might reduce the hyperhomocysteinaemia [23, 24]. However, the supply of folate coenzymes in vivo depends primarily on the quantity and bioavailability of ingested folic acid/folate and the rate of loss by urinary and fecal routes and through catabolism. Additionally, folate is highly susceptible to oxidative destruction. In fact, $50-95 \%$ of folate content in food is estimated to be lost during storage, preparation, or manufacturing processes [25].

The aim of this work was to design and evaluate zein nanoparticles as carriers capable of improving the bioavailability of folic acid when orally administered. For this purpose, these zein nanoparticles were prepared by an original procedure and their capability to improve the oral bioavailability of folic acid was evaluated and compared with a conventional aqueous solution of the vitamin in rats.

\section{Materials and methods Materials}

Zein, folic acid, lysine, arginine, pepsin, pancreatin, mannitol and sodium chloride were purchased from Sigma-Aldrich (Steinheim, Germany). Ethanol, acetonitrile and o-phosphoric acid (HPLC grade) were obtained from Merck (Darmstadt, Germany). Perylene-Red (BASF Lumogen® F Red 305; Lumogen red) was from Kremer Pigments Inc. (Aichstetten, Germany) and Tissue-Tek ${ }^{\circledR}$ OCT compound from Sakura Finetek Europe (Alphen, The Netherlands). 4',6diamidino-2-phenylindole (DAPI) was obtained from Biotium Inc. (Hayward, CA). lodine 125 was from Perkin Elmer (USA). AccuDiag ${ }^{\text {TM }}$ Folate-Folic acid ELISA Kit was purchased from Diagnostic Automation/Cortez Diagnostics Inc. (USA). Deionized water (18.2 $\mathrm{M} \Omega$ resistivity) was prepared by a water purification system (Wasserlab, Spain). All reagents and chemicals used were of analytical grade.

\section{Preparation of zein nanoparticles}

Zein nanoparticles were prepared by a desolvation procedure followed by a purification step by ultrafiltration and subsequent drying in a spray-drier apparatus.

\section{Empty zein nanoparticles (NP-Z)}

Briefly, $600 \mathrm{mg}$ zein and $100 \mathrm{mg}$ lysine were firstly dissolved in $70 \mathrm{~mL}$ of a mixture of ethanol and water (1:1 by vol.) under magnetic stirring at room temperature. Then, nanoparticles were obtained by the continuous addition of $70 \mathrm{~mL}$ purified water. The suspension was purified and concentrated by ultrafiltration using a membrane cartridge with a $50 \mathrm{kDa}$ pore size polysulfone (Medica SPA, Italy). Finally, $20 \mathrm{~mL}$ of an aqueous solution of mannitol (100 $\mathrm{mg} / \mathrm{mL}$ ) was added to the suspension of zein nanoparticles and the mixture was dried in a Büchi Mini Spray Drier B-290 apparatus (Büchi Labortechnik AG, Switzerland) under the following experimental conditions: (i) inlet temperature: 
$90^{\circ} \mathrm{C}$, (ii) outlet temperature: $45-50^{\circ} \mathrm{C}$, (iii) air pressure: 5 bar, (iv) pumping rate:

\section{$5 \mathrm{~mL} / \mathrm{min},(\mathrm{v})$ aspirator of $100 \%$ and (vi) air flow: $900 \mathrm{~L} / \mathrm{h}$.}

\section{Folic acid-loaded zein nanoparticles (FA-NP-Z)}

The preparation of zein nanoparticles loaded with folic acid (FA-NP-Z) was similar to that of the empty particles, with some minor adjustments. For this purpose, $600 \mathrm{mg}$ zein and $100 \mathrm{mg}$ lysine were dissolved in $70 \mathrm{~mL}$ of a mixture of ethanol and water (1:1 by vol.). In parallel, $200 \mathrm{mg}$ folic acid was dissolved in $50 \mathrm{~mL}$ of an aqueous solution of lysine $(4 \mathrm{mg} / \mathrm{mL})$. Then, $15 \mathrm{~mL}$ of the aqueous folic acid solution were added to the zein solution and the resulting mixture was incubated at room temperature for 10 min under magnetic stirring. Finally, zein nanoparticles were obtained by the addition of $70 \mathrm{~mL}$ purified water. The suspension was purified and dried as described above.

\section{Characterization of nanoparticles Size, zeta potential and morphology}

The mean hydrodynamic diameter and the zeta potential of the nanoparticles were determined by photon correlation spectroscopy (PCS) and electrophoretic laser Doppler anemometry, respectively, using a Zetaplus apparatus (Brookhaven Instrument Corporation, USA). The diameter of the nanoparticles was determined after dispersion in distilled water (1:10) and was measured at $25^{\circ} \mathrm{C}$ with a scattering angle of $90^{\circ}$. The zeta potential was measured after dispersion of the dried nanoparticles in $1 \mathrm{mM} \mathrm{pH} 6 \mathrm{KCl}$ solution.

The morphology and shape of nanoparticles was examined using a field emission scanning electron microscope FE-SEM (ULTRA Plus, Zeiss, The Netherlands). Prior to analysis, particles were washed to remove mannitol. For this purpose, spray-dried nanoparticles were resuspended in distilled water and centrifuged at $17,000 \times \mathrm{g}$ for $10 \mathrm{~min}$. Then, the supernatants were discarded and the obtained pellets were mounted on copper grids. Finally, the pellet was shaded with an amalgam of gold/palladium during fifteen seconds using a sputter coater (K550X Emitech, Ashford, UK).

\section{Yield of the preparative process}

In order to quantify the amount of protein transformed into nanoparticles, $10 \mathrm{mg}$ of the nanoparticle formulation were dispersed in water and centrifuged at $17,000 \times \mathrm{g}$ for $20 \mathrm{~min}$. Supernatants were discarded and the pellets were digested with ethanol $75 \%$. Then, the amount of protein was quantified by UV spectrophotometry at $278 \mathrm{~nm}$ in an Agilent 8453 system (Agilent Technologies, USA). For analysis, calibration curves were constructed between 90 and 1200 $\mu \mathrm{g} / \mathrm{mL}\left(R^{2}>0.999\right.$; quantification limit $\left.=143 \mu \mathrm{g} / \mathrm{mL}\right)$.

The amount of protein forming nanoparticles in the formulation was estimated as the ratio between the amount of the protein quantified in the pellet and the total amount of zein used for the preparation of nanoparticles.

\section{Folic acid analysis}

The amount of folic acid loaded into the nanoparticles was quantified by HPLCUV using a previously described analytical method [26] with minor modifications. Analyses were carried out in an Agilent model 1100 Series LC 
System coupled to a diode-array detector set at $290 \mathrm{~nm}$. The data were analyzed using ChemStation G2171 v. B.01.03 software (Agilent, USA). The chromatographic system was equipped with a reverse C18 Alltima column (150 $\mathrm{mm} \times 2.1 \mathrm{~mm}$, particle size $5 \mu \mathrm{m}$; Altech, USA) and a Gemini C18 precolumn (particle size $5 \mu \mathrm{m}$; Phenomenex, CA, USA). The mobile phase, pumped at $0.25 \mathrm{~mL} / \mathrm{min}$, was a mixture of phosphoric acid $(33 \mathrm{mM}, \mathrm{pH} 2.3)$ and acetonitrile under gradient conditions [26]. The column was heated to $40^{\circ} \mathrm{C}$ and the injection volume was $10 \mu \mathrm{L}$. Under these conditions, folic acid eluted at $21.2 \pm$ $0.5 \mathrm{~min}$. Calibration curves were designed over the range of 2 and $200 \mu \mathrm{g} / \mathrm{mL}$ $\left(R^{2}>0.999\right)$. The limit of quantification was calculated to be $4.3 \mu \mathrm{g} / \mathrm{mL}$.

For analysis, $10 \mathrm{mg}$ nanoparticles was dispersed in $1 \mathrm{~mL}$ water and centrifuged. The amount of encapsulated folic acid was calculated by dissolution of the pellets in $75 \%$ ethanol $(1 \mathrm{~mL})$. In parallel, the total amount of folic acid in the dry formulations was quantified by direct digestion of $10 \mathrm{mg}$ formulation with $1 \mathrm{~mL}$ ethanol $75 \%$. In all cases the samples were filtered through $0.45 \mu \mathrm{m}$ membranes before analysis. Each sample was assayed in triplicate and the results are expressed as the amount of resveratrol $(\mu \mathrm{g})$ per $\mathrm{mg}$ of nanoparticles. The encapsulation efficiency (EE), expressed in percentage, was calculated as the ratio between the amount of folic acid quantified in the pellets and the total amount of folic acid quantified in the dry powder.

\section{In vitro release study}

Release experiments were conducted under sink conditions at $37^{\circ} \mathrm{C}$ using simulated gastric fluid (SGF; pH 1.2; pepsin $0.32 \% \mathrm{w} / \mathrm{v}$ ) and intestinal fluid (SIF; $\mathrm{pH}$ 6.8; pancreatin $1 \% \mathrm{w} / \mathrm{v}$ ). The studies were performed under agitation in a Vortemp $56^{\mathrm{TM}}$ Shaking Incubator (Labnet International Inc., NJ, USA) after the dispersion of the nanoparticles in the appropriate medium.

For each specific time interval, $20 \mu \mathrm{g}$ folic acid formulated in nanoparticles were resuspended in $1 \mathrm{~mL}$ of the corresponding simulated fluid. The different formulations were kept in the SGF for 2 hours before being transferred to SIF. At different intervals, samples were collected and centrifuged at $17,000 \mathrm{rpm}$ for 20 minutes. The amount of folic acid released was quantified by HPLC from the supernatants as described above.

\section{Zein nanoparticles labelling}

Radiolabelling of zein nanoparticles ( ${ }^{125}$ I-NP-Z)

Zein nanoparticles were radiolabelled with lodine-125 $\left({ }^{125} \mathrm{INa}\right)$ by standard mild oxidative iodination. For this purpose, $10 \mathrm{mg}$ empty zein nanoparticles were tagged with 2 iodobeads and $3.5 \mu \mathrm{L}$ of ${ }^{125} \mathrm{INa}$ in $600 \mu \mathrm{L}$ of a mixture between PBS and water for injection (1:2 by vol). After $15 \mathrm{~min}$ of incubation, iodine zein nanoparticles $\left({ }^{125}\right.$ I-NP-Z) were obtained.

The stability of the radiolabelling was evaluated by TLC. For this purpose, ${ }^{125} \mid-$ NP-Z in dialysis cassettes were introduced in aqueous media and the presence of free iodide was revealed by TLC.

\section{Lumogen red loaded in zein nanoparticles (LR-NP-Z)}

Zein nanoparticles were fluorescently labelled with Lumogen® F Red 305 (LR$\mathrm{NP}-\mathrm{Z})$. Briefly, $2 \mathrm{mg}$ Lumogen $\otimes$ red in acetone $(5 \mathrm{~mL})$ were added to the 
hydroalcoholic solution of zein and lysine. Then, zein nanoparticles were formed by the addition of $70 \mathrm{~mL}$ purified water. The resulting nanoparticles were purified and dried under the same conditions described above. The amount of Lumogen ${ }^{\circ} \mathrm{F}$ Red 305 was determined by colorimetry at wavelength $540 \mathrm{~nm}$ in a spectrophotometer Agilent 8453 system (USA). For this purpose, $10 \mathrm{mg}$ of the formulations were re-suspended in purified water and centrifuged at $17,000 \mathrm{rpm}$ for $20 \mathrm{~min}$. Pellets were then dissolved in ethanol $75 \%$. For quantification, standard curves of Lumogen red in ethanol $75 \%$ were used (concentration range of $5-30 \mu \mathrm{g} / \mathrm{mL} \mathrm{R}^{2} \geq 0.999$ ).

Prior the use of fluorescently labelled nanoparticles for in vivo studies, the stability of the marker in the nanoparticles was assessed by incubation in simulated gastric $(\mathrm{pH} \mathrm{1.2)}$ and intestinal $(\mathrm{pH} \mathrm{6.8)}$ fluids.

\section{In vivo distribution study} (Barcelona, Spain) and the protocols were approved by the Ethical Committee for Animal Experimentation of the University of Navarra (protocol number 11712 and 059-13). Prior to the experiment, animals were placed in metabolic cages and drink provided ad libitum.

For radiolabelled nanoparticles, animals $(200-250 \mathrm{~g})$ received a $1 \mathrm{~mL}$ single dose of an aqueous suspension of nanoparticles (10 mg of ${ }^{125} \mathrm{I}-\mathrm{NP}-\mathrm{Z}$ ). As control, an aqueous suspension of ${ }^{125} \mathrm{I}$ was administered by oral route. Animals were anesthetized with isofluorane and place in prone position on the gammacamera. The gammagraphic studies were performed in a E.cam DualHead-Variable-Angle System gammacamera (Siemens Medical Systems, USA) The images were obtained 2, 24 and 48 hours after the administration of the radiolabelled nanoparticles.

For fluorescently labelled nanoparticles, a protocol previously described was used [27]. Thus, the animals received orally a single dose of $30 \mathrm{mg}$ nanoparticles (LP-NP-Z) dispersed in $1 \mathrm{~mL}$ water. Two hours later, the animals were sacrificed and guts were removed. Jejunum portions of $1 \mathrm{~cm}$ were collected, cleaned with PBS, stored in the tissue proceeding medium O.C.T. and frozen at $-80^{\circ} \mathrm{C}$. Each portion was then cut into $5-\mu \mathrm{m}$ sections on a cryostat and attached to glass slides. Finally, these samples were fixed with formaldehyde and incubated with DAPI (4',6-diamidino-2-phenylindole) for 15 minutes before the cover assembly. The presence of both fluorescently loaded poly(anhydride) nanoparticles in the intestinal mucosa and the cell nuclei dyed with DAPI were visualized in a fluorescence microscope (Axioimager M1, Zeiss) with a coupled camera (Axiocam ICc3, Zeiss) and fluorescent source (HBO 100 , Zeiss. The images were captured with the software ZEN (Zeiss).

\section{In vivo pharmacokinetic studies in male Wistar rats Pharmacokinetic studies}

Pharmacokinetic studies were performed in male Wistar rats (200-250 g) obtained from Harlan (Barcelona, Spain). Studies were approved by the Ethical Committee for Animal Experimentation of the University of Navarra (protocol number 014-10) in accordance with the European legislation on animal experiments. Prior to the experiment, animals were adaptively fed for 1 week 
with free access to a Folic Acid deficient diet (TD 95247, Harlan, USA) and drinking water $\left(22 \pm 2^{\circ} \mathrm{C}\right.$; 12-h light and 12-h dark cycles; $50-60 \%$ relative humidity). Previous to the oral administration of the formulations, animals were fasted overnight to avoid interference with the absorption, allowing free access to water.

For the pharmacokinetic study, rats were randomly divided into 4 groups of 6 animals each. The experimental groups were an aqueous solution of folic acid extemporaneously prepared (FA dissolved in PBS) and folic acid-loaded zein nanoparticles (FA-NP-Z) dispersed in water. As controls, a group of animals was intravenously administered with a solution of folic acid in PBS and the last group of rats received PBS (without folic acid) orally. The single folic acid administered dose was $1 \mathrm{mg} / \mathrm{kg}$ body weight either orally with a blunt needle via the oesophagus into the stomach or intravenously via tail vein.

Blood samples were collected at set times after administration in specific serum tubes (SARSTEDT Microtube $1,1 \mathrm{~mL}$ Z-Gel). Volemia was recovered intraperitoneally with an equal volume of sterile saline solution pre-heated to body temperature. Samples were immediately centrifuged at 10,000 rpm for 10 min. Serum was separated into clean tubes and kept frozen at $-80{ }^{\circ} \mathrm{C}$ until analysis.

\section{Determination of folic acid in serum}

The amount of folic acid in serum was determined by an Enzyme Immunoassay. Calibrator and quality control samples were prepared by adding appropriate volumes of standard folic acid solution in PBS to serum. Calibration curves were designed over the range $4-450 \mathrm{ng} / \mathrm{mL}\left(R^{2}>0.996\right)$. For analysis, $100 \mathrm{~mL}$ of the serum samples were added to each well of the microtiter plate, followed by the addition of $50 \mathrm{~mL}$ folic acid antibody. After incubation for $60 \mathrm{~min}$ at room temperature, the plate was washed three times with the washing solution (PBS-Tween 20 0.5\%). Then, $100 \mathrm{~mL}$ conjugate (anti-mouse-lgG-HRP) was added into each well and after $60 \mathrm{~min}$ at room temperature, the plate was washed again for three times with the washing solution. For the reaction, 100 $\mathrm{mL}$ of substrate was added into each well and incubated in the dark for $20 \mathrm{~min}$ at room temperature. The reaction was stopped by the addition of $100 \mathrm{~mL}$ sulphuric acid $0.5 \mathrm{M}$ into each well. Finally, the absorbance was measured at $450 \mathrm{~nm}$ in an ELISA reader (Labsystems iEMS Reader MF).

Under these experimental conditions, the limit of quantification of this method was calculated to be $4 \mathrm{ng} / \mathrm{mL}$. The recovery of folic acid from serum samples was $90.1 \pm 0.3 \%$. Accuracy values during the same day (intraday assay) at low, medium and high concentrations of FA were always within the acceptable limits (less than 15\%).

\section{Pharmacokinetic data analysis}

The pharmacokinetic analysis of serum concentration plotted against time data was performed using a non-compartmental model with the WinNonlin 5.2 software (Pharsight Corporation, Mountain View, USA). The following parameters were estimated: maximal serum concentration $\left(\mathrm{C}_{\max }\right)$, time taken to reach $C_{\max }\left(T_{\max }\right)$, area under the concentration-time curve from time 0 to $\infty$ $(A U C)$, mean residence time (MRT), clearance $(\mathrm{Cl})$, volume of distribution $(\mathrm{V})$ 
and half-life in the terminal phase $\left(t_{1 / 2}\right)$. Furthermore, the relative oral bioavailability ( $\mathrm{Fr}$, expressed in percentage) of folic acid was estimated as the ratio between the areas under the curve for the oral $\left(A U C_{\text {oral }}\right)$ and intravenous

\section{Results}

\section{Folic acid loaded zein nanoparticles}

Table 1 summarizes the main physicochemical properties of folic acid-loaded nanoparticles. When folic acid was encapsulated into zein nanoparticles, a moderate increase in the mean size of the resulting carriers was observed (about $164 \mathrm{~nm}$ for empty nanoparticles vs $193 \mathrm{~nm}$ for FA-NP-Z); whereas the negative zeta potential decreased from $-46 \mathrm{mV}$ (control nanoparticles) to -30 $\mathrm{mV}$ (folic acid-loaded nanoparticles). The folic acid loading into the zein nanoparticles (FA-NP-Z) was calculated to be around $54 \mu \mathrm{g} / \mathrm{mg}$ nanoparticle, with encapsulation efficiency close to $57 \%$.

The morphological analysis by scanning electron microscopy (Figure 1) showed that folic acid-loaded zein nanoparticles consisted of homogeneous populations of spherical nanoparticles with a smooth surface and an apparent similar size to that obtained by photon correlation spectroscopy.

\section{Table 1}

\section{Figure 1}

\section{In vitro release study}

Figure 2 represents the release profile of folic acid from the zein nanoparticles formulations as cumulative percentage of the vitamin released as a function of time. When nanoparticles were incubated in SGF, no release of folic acid was observed. On the contrary, when zein nanoparticles were assayed in SIF, the release of folic followed a profile characterized by two different steps. In the first one, approximately $70 \%$ of the loaded folic acid was rapidly released. Then, after this burst effect, the remaining vitamin was released in a sustained way up to the end of the experiment (24 hours).

Figure 2 
In vivo distribution study of ${ }^{125} \mathrm{I}-\mathrm{NP}-\mathrm{Z}$ and Lumogen-NP-Z in the gut mucosa

Figure 3 shows the biodistribution (SPECT-CT images) of free 125-iodine (Figure 3A) and zein nanoparticles radiolabelled with 125-iodine (Figure 3B) orally administered to rats. radioactivity was always found in their stomach and thyroid. On the other hand, the radioactivity associated to zein nanoparticles was visualized in the stomach 2 hours after administration; although, twenty two hours later the radioactive signal was also found at the thyroid and the distal areas of the colon. Finally, 48 hours after administration, the remaining activity was observed in the thyroid and stomach of animals, in which the signal was significantly lower than that observed at the previous times. The activity in the thyroid demonstrates in vivo physiologic de-iodination of iodine labelled nanoparticles.

\section{Figure 3}

Figure 4 shows fluorescence microscopy images of jejunum samples of animals treated with Lumogen $\circledast$ red formulations. Control formulation (an aqueous suspension of the fluorescent marker) was observed in the lumen of the small intestine of animals as large aggregates (Figure 4A) and no fluorescence was visualized in the vicinity of the intestinal epithelium (Figure 4B). On the contrary, when the fluorescent marker was encapsulated in zein nanoparticles, fluorescence appeared to be in the protective mucus layer, covering the surface of the intestinal epithelium (Figures 4C and 4D).

\section{Figure 4}

\section{Pharmacokinetic studies in Wistar rats}

When folic acid was administered orally as aqueous solution, the levels of the vitamin in the sera of animals increased rapidly during the first $1 \mathrm{~h}$ postadministration, in which the $\mathrm{C}_{\max }$ was reached (Figure 5). Then, the vitamin levels decreased slowly until the end of the experiment $(24 \mathrm{~h}$ postadministration). For the formulation based on zein nanoparticles, the levels of folic acid in the sera of animals displayed a similar profile to that observed for the free folic acid (FA solution). However, the serum levels of the vitamin from nanoparticles were significantly higher than those observed for the aqueous solution of folic acid.

\section{Figure 5}

Table 2 summarizes the pharmacokinetic parameters derived from the analysis of the data obtained after the administration of the different folic acid formulations to rats. When folic acid was administrated orally as aqueous solution, the AUC was $1.4 \mu \mathrm{g} \mathrm{h} / \mathrm{mL}$; whereas, this parameter was $3.0 \mu \mathrm{g} \mathrm{h} / \mathrm{mL}$ when the vitamin was given after its encapsulation in zein nanoparticles. Similarly, the peak plasma concentration $\left(C_{\max }\right)$ of folic acid in the nanoparticles 
was around 2- times higher than for the vitamin aqueous solution. On the contrary, other important pharmacokinetic parameters of folic acid (e.g., volume of distribution, clearance or half-life of the terminal phase) were similar when the vitamin was administered as aqueous solution or loaded in zein nanoparticles. Finally, the relative oral bioavailability of folic acid when incorporated in zein nanoparticles was of about $70 \%$, whereas for the folic acid aqueous solution the oral bioavailability was only of $35 \%$.

\section{Table 2}

\section{Discussion}

Folic acid, as other weak acid compounds, possesses a pH-dependent aqueous solubility, being insoluble in aqueous media below pH 5 [28]. In vivo, the $\mathrm{pH}$ of the stomach contents may induce the precipitation of the vitamin in macroscopic aggregates that, once in the small intestine ( $\mathrm{pH}$ around 5-6), would be (at least in part) re-dissolved. However, in these $\mathrm{pH}$ conditions, and because of the hydrophilic nature of the charged molecule, specific transporters are required for folic acid absorption. These highly specific transporters (the reduced folate carrier, RFC, and the proton-coupled folate transporter, PCFT) are expressed at the apical brush-border membrane of the proximal jejunum [29] in which the absorption of the vitamin takes place [30].

On the other hand, zein is a biodegradable and biocompatible material, economic to use and with a "GRAS" status [31]. In addition, zein is an amphiphilic protein with an important ability to interact with solutes like drugs [32] or amino acids [33]. Moreover, zein displays mucoadhesive properties [12] and a relatively high resistance to the effect of digestive enzymes [3].

In this work, zein nanoparticles were prepared under mild conditions by a desolvation technique after the addition of water to a hydroalcoholic solution of the protein and lysine. Then nanoparticles were purified and, finally, dried in a spray-drier apparatus. The presence of lysine was necessary to facilitate the redispersion of the dry powder in water by simple manual agitation. Under these experimental conditions, folic acid-loaded zein nanoparticles displayed a mean size after reconstitution of about $200 \mathrm{~nm}$ with a negative zeta potential of -24 $\mathrm{mV}$ and a low polydispersity index (Table 1). The folic acid content was close to $54 \mathrm{\mu g} / \mathrm{mg}$ nanoparticles, which is approximately 3-times higher than the payload reported by Perez-Masiá and collaborators who used nano- and microcapsules from either whey protein or starch [34].

Interestingly, the release of folic acid from zein nanoparticles was found to be dependent of the $\mathrm{pH}$ conditions. Thus, under simulated gastric conditions, folic acid was not released from zein nanoparticles. Nevertheless, when nanoparticles were incubated in SIF, a burst effect of approximately $70 \%$ of the folic acid content was observed (Figure 2). These findings would be directly related with the fact that the solubility of folic acid in water is dependent on its ionization (pKa values of $4.65,6.75$ and $9[35,36]$ ). In SIF, the two carboxylic acid groups of folic acid would be deprotonated, resulting in a negative net charge similar to that observed for zein nanoparticles. Thus the repulsion between ionized folic acid and zein would result in a rapid release of the vitamin from the nanoparticles. On the other hand, after this burst effect of folic acid in 
SIF, the remaining vitamin (around $30 \%$ ) was slowly released till the end of the experiment (Figure 2). This fact might be related with the capability of folic acid to bind to proteins, such as albumins [37] and caseins [38], through hydrogen and hydrophobic bonds. To the best of our knowledge, there is no information suggesting the presence of binding sites for folic acid in zein; however, in accordance with the release data, we can hypothesize that a fraction of the loaded folic acid would be stabilized within the protein matrix by non-covalent binding interactions. Therefore, the remaining fraction would be released slowly during the degradation of the nanoparticles.

In the present study, the relative oral bioavailability of folic acid formulated as aqueous solution was calculated to be $35 \%$. The highest serum concentration of folic acid occurred 1 hour after the administration, and values returned to baseline after 24 hours. These results are in line with previous data reported in the literature by other research groups [39, 40]. On the other hand, zein nanoparticles provided higher folic acid levels than the aqueous solution. As a consequence, the relative oral bioavailability of folic acid when administered after its encapsulation in zein nanoparticles was calculated to be close to $70 \%$ and 2-times higher than when administered as oral solution (Table 2). Other important aspects to highlight are that both the serum curve profiles (Figure 5) and the primary pharmacokinetic parameters (volume of distribution, clearance, serum half-life) of folic acid were independent on the formulation tested (aqueous solution and zein nanoparticles). Thus, the differences in the oral bioavailability of the vitamin might only be due to the capabilities of zein nanoparticles to both give protection against the macroscopic aggregation by precipitation of the vitamin in acidic conditions and act as carriers to transport the folic acid to the absorptive membrane.

When orally administered, zein nanoparticles remained within the gastrointestinal tract for a period of at least $24 \mathrm{~h}$ post-administration (Figure 3 ). The absence of signals in the liver, spleen and lungs of animals suggested that zein nanoparticles were not capable of entering into the circulation from the gut (Figure 3). Within the gastrointestinal tract of animals, and in accordance with data from fluorescently labelled nanoparticles, these carriers would be capable of interacting with the mucus layer protecting the epithelium surface (Figures $4 \mathrm{C}$ and 4D). This last observation would be in line with previous data suggesting the mucoadhesive properties of zein [12, 41, 42].

To sum up, zein nanoparticles would be capable of transporting the cargo to the small intestine. Once there, the mucoadhesive properties of zein would be responsible for an increase in the residence time of these carriers in the upper region of the gastrointestinal tract, in which the absorption of folic acid is favored $[29,30]$.

\section{Conclusions}

Zein nanoparticles offer adequate properties for oral delivery purposes. Orally administered, these nanoparticles are localized within the gut in close contact with the gut mucosa. Regarding folic acid, its encapsulation in zein nanoparticles improved its relative oral bioavailability about 2-fold when compared with an aqueous solution of the vitamin. This fact would be related 
with the capability of these nanoparticles to reach the small intestine mucosa and develop mucoadhesive interactions.

\section{Acknowledgements}

This work was supported by the Regional Government of Navarra (Alimentos Funcionales, Euroinnova call) and the Spanish Ministry of Science and Innovation and Gobierno de Navarra (ADICAP; ref. IPT-2011-1717-900000). Rebeca Penalva acknowledges the "Asociación de Amigos Universidad de Navarra" for the financial support.

\section{References}

[1] T.J. Anderson, B.P. Lamsal, Zein extraction from corn, corn products, and coproducts and modifications for various applications: a review, Cereal Chem. 88 (2011) 159-173.

[2] D. Fu, C.L. Weller, R.L. Wehling, Zein: properties, preparations, and applications, Food Sci. Biotechnol. 8 (1999) 1-10.

[3] R. Shukla, M. Cheryan, Zein: the industrial protein from corn, Ind. Crop. Prod. 13 (2001) 171-192.

[4] A. Esen, A proposed nomenclature for the alcohol-soluble proteins (zeins) of maize (Zea mays L.), J. Cereal Sci. 5 (1987) 117-128.

[5] F.A. Momany, D.J. Sessa, J.W. Lawton, G.W. Selling, S. Hamaker, J.L. Willett, Structural characterization of a-zein, J. Agric. Food Chem. 54 (2006) 543-547.

[6] G.W. Selling, A. Biswas, A. Patel, D.J. Walls, C. Dunlap, Y. Wei, Impact of solvent on electrospinning of zein and analysis of resulting fibers, Macromol. Chem. Physic. 208 (2007) 1002-1010.

[7] T. Padgett, I. Han, P. Dawson, Incorporation of food-grade antimicrobial compounds into biodegradable packaging films, J. Food Protect. 61 (1998) 1330-1335.

[8] H. Guo, Y. Shi, A novel zein-based dry coating tablet design for zero-order release, Int. J. Pharm. 370 (2009) 81-86.

[9] H.X. Guo, J. Heinämäki, J. Yliruusi, Stable aqueous film coating dispersion of zein, J. Colloid Interface Sci. 322 (2008) 478-484.

[10] J. Tu, H. Wang, H. Li, K. Dai, J. Wang, X. Zhang, The in vivo bone formation by mesenchymal stem cells in zein scaffolds, Biomaterials 30 (2009) 4369-4376.

[11] Y. Luo, Z. Teng, Q. Wang, Development of zein nanoparticles coated with carboxymethyl chitosan for encapsulation and controlled release of vitamin D3, J. Agric. Food Chem. 60 (2012) 836-843.

[12] A. Patel, Y. Hu, J.K. Tiwari, K.P. Velikov, Synthesis and characterisation of zein-curcumin colloidal particles, Soft Matter 6 (2010) 6192-6199.

[13] D. Xiao, P.M. Davidson, Q. Zhong, Spray-dried zein capsules with coencapsulated nisin and thymol as antimicrobial delivery system for enhanced antilisterial properties, J. Agric. Food Chem. 59 (2011) 7393-7404.

[14] R. Paliwal, S. Palakurthi, Zein in controlled drug delivery and tissue engineering, J. Control. Release 189 (2014) 108-122. 
[15] T. Zou, Z. Li, S.S. Percival, S. Bonard, L. Gu, Fabrication, characterization, and cytotoxicity evaluation of cranberry procyanidins-zein nanoparticles, Food Hydrocolloid 27 (2012) 293-300. [16] G. Kronenberg, M. Colla, M. Endres, Folic acid, neurodegenerative and neuropsychiatric disease, Curr. Mol. Med. 9 (2009) 315-323.

[17] R. lyer, S. Tomar, Folate: a functional food constituent, J. Food Sci. 74 (2009) R114-R122.

[18] E.H. Reynolds, The neurology of folic acid deficiency, Handb. Clin. Neurol. 120 (2014) 927-943.

[19] M. Lövdén, L. Bergman, R. Adolfsson, U. Lindenberger, L. Nilsson, Studying individual aging in an interindividual context: typical paths of agerelated, dementia-related, and mortality-related cognitive development in old age, Psychol. Aging 2 (2005) 303-316.

[20] J. Sarris, N. Schoendorfer, D.J. Kavanagh, Major depressive disorder and nutritional medicine: a review of monotherapies and adjuvant treatments, Nutr. Rev. 67 (2009) 125-131.

[21] R.T. Owen, Folate augmentation of antidepressant response, Drugs Today (Barc) 49 (2013) 791-798.

[22] R. Zeng, C. Xu, Y. Xu, Y. Wang, M. Wang, The effect of folate fortification on folic acid-based homocysteine-lowering intervention and stroke risk: a metaanalysis, Public Health Nutr. (2014) 1-8.

[23] A. de Bree, W.M. Verschuren, A.L. Bjorke-Monsen, N.M. van der Put, S.G. Heil, F.J. Trijbels, H.J. Blom, Effect of the methylene tetrahydrofolate reductase $677 \mathrm{C}-->\mathrm{T}$ mutation on the relations among folate intake and plasma folate and homocysteine concentrations in a general population sample, Am. J. Clin. Nutr. 77 (2003) 687-693.

[24] S.E. Chiuve, E.L. Giovannucci, S.E. Hankinson, D.J. Hunter, M.J. Stampfer, W.C. Willett, E.B. Rimm, Alcohol intake and methylene tetrahydrofolate reductase polymorphism modify the relation of folate intake to plasma homocysteine, Am. J. Clin. Nutr. 82 (2005) 155-162.

[25] M.K. Off, A.E. Steindal, A.C. Porojnicu, A. Juzeniene, A. Vorobey, A. Johnsson, J. Moan, Ultraviolet photodegradation of folic acid, J. Photochem. Photobiol B. 80 (2005) 47-55.

[26] R. Penalva, I Esparza, M. Agüeros, C. Gonzalez-Nvarro, C. GonzalezFerrero, J.M. Irache, Casein nanoparticles as carriers for the oral delivery of folic acid, Food Hydrocolloid. 44 (2015) 399-406.

[27] L. Inchaurraga, N. Martín-Arbella, V. Zabaleta, G. Quincoces, I. Peñuelas, J.M. Irache, In vivo study of the mucus-permeating properties of PEG-coated nanoparticles following oral administration, Eur. J. Pharm. Biopharm. (in press) [28] Z. Wu, X. Li, C. Hou, Y. Qian, Solubility of folic acid in water at $\mathrm{pH}$ values between 0 and 7 at temperatures $(298.15,303.15$, and 313.15) K, J. Chem. Eng. Data 55 (2010) 3958-3961.

[29] R. Zhao, L.H. Matherly, I.D. Goldman, Membrane transporters and folate homeostasis: intestinal absorption and transport into systemic compartments and tissues, Exp. Rev. Mol. Med. 11 (2009) e4 (doi: 10.1017/S1462399409000969). 
624 [30] V.S. Subramanian, J.S. Marchant, H.M. Said, Apical membrane targeting 625 and trafficking of the human proton-coupled transporter in polarized epithelia, 626 Am. J. Physiol. Cell Physiol. 294 (2008) C233-240.

627 [31] P. Hurtado-Lopez, S. Murdan, Formulation and characterisation of zein 628 microspheres as delivery vehicles, J. Drug Del. Sci. Technol. 15 (2005) 267629272.

630 [32] F. Sousa, A. Luzardo-Álvarez, J. Blanco-Méndez, M. Martín-Pastor, NMR 631 techniques in drug delivery: application to zein protein complexes, Int. J. Pharm. 632439 (2012) 41-48.

633 [33] T. Cserhati, E. Forgacs, Effect of pH and salts on the binding of free amino 634 acids to the corn protein zein studied by thin-layer chromatography, Amino 635 Acids 28 (2005) 99-103.

636 [34] R. Pérez-Masiá, R. López-Nicolás, M.J. Periago, G. Ros, J.M. Lagaron, A. 637 López-Rubio, Encapsulation of folic acid in food hydrocolloids through 638 nanospray drying and electrospraying for nutraceutical applications, Food 639 Chem. 168 (2015) 124-133.

640 [35] M.J. ONeil, The Merck Index: An Encyclopedia of Chemicals, Drugs, and 641 Biologicals, 14th ed., People's Health Publishing House, Beijing, 2006.

642 [36] A.R. Patel, E.C. Bouwens, K.P. Velikov, Sodium caseinate stabilized zein 643 colloidal particles, J. Agric. Food Chem. 58 (2010) 12497-12503.

644 [37] P. Bourassa, H.A. Tajmir-Riahi, Locating the binding sites of folic acid with 645 milk $\alpha$ - and $\beta$-caseins, J. Phys. Chem. B. 116 (2012) 513-519.

646 [38] P. Bourassa, I. Hasni, H.A. Tajmir-Riahi, Folic acid complexes with human 647 and bovine serum albumins, Food Chem. 129 (2011) 1148-1155.

648 [39] N.C. Alemdaroglu, U. Dietz, S. Wolffram, H. Spahn-Langguth, P. Langguth, 649 Influence of green and black tea on folic acid pharmacokinetics in healthy 650 volunteers: potential risk of diminished folic acid bioavailability, Biopharm. Drug 651 Dispos. 29 (2008) 335-348.

652 [40] P. Nguyen, R. Boskovic, P. Yazdani, B. Kapur, H. Vandenberghe, G. 653 Koren, Comparing folic acid pharmacokinetics among women of childbearing 654 age: single dose ingestion of 1.1 versus $5 \mathrm{mg}$ folic acid, Can. J. Clin. 655 Pharmacol. 15 (2008) 314-322.

656 [41] S. Wongsasulak, S. Pathumban, T. Yoovidhya, Effect of entrapped a657 tocopherol on mucoadhesivity and evaluation of the release, degradation, and 658 swelling characteristics of zein-chitosan composite electrospun fibers, J. Food 659 Eng. 120 (2014) 110-117.

660 [42] B. Zhang, Y. Luo, Q. Wang, Effect of acid and base treatments on 661 structural, rheological, and antioxidant properties of a-zein, Food Chem. 124 662 (2011) 210-220. 


\section{Figure Captions}

Figure 1. Scanning electron microscopy (SEM) microphotographs obtained from folic acid-loaded zein nanoparticles.

Figure 2. Folic acid release profile from zein nanoparticles after incubation in simulated gastric fluid $(0-2 \mathrm{~h}$ ) and intestinal fluid $(2-48 \mathrm{~h})$ under sink conditions. Data are expressed as the mean $\pm S D, n=3$.

Figure 3. Biodistribution of 125-lodine control and radiolabelled zein nanoparticles. Panels $A$ and $B$ show gammacamera images after oral administration of 125 -lodine $(3 \mathrm{~A})$ and $10 \mathrm{mg}{ }^{125} \mathrm{I}-\mathrm{NP}-\mathrm{Z}(3 \mathrm{~B})$ at 2,24 and 48 hours post administration.

Figure 4. Fluorescence microscopy images of jejunum samples 2 hours after the oral administration of either a Lumogen $\otimes$ red aqueous suspension ( $A$ and $B$ ) or zein nanoparticles fluorescently labelled with Lumogen $®$ red. Nuclei of cells were stained blue with DAPI.

Figure 5. Folic acid serum concentration vs time after a single oral administration of $1 \mathrm{mg} / \mathrm{kg}$ for the different formulations tested. i) Folic acid solution in PBS (•; FA sol), ii) Folic acid loaded in zein nanoparticles ( $\mathbf{\Delta}$; FANP-Z). Data expressed as the mean \pm SD; $(n=6)$. 
693

694

695

696

697

698

699

700

701

702

703

\section{Tables}

Table 1. Physicochemical characterization of zein nanoparticles. NP-Z: empty nanoparticles; FA: folic acid; FA-NP-Z: folic acid-loaded zein nanoparticles. Data expressed as mean $\pm S D, n=3$.

\begin{tabular}{|c|c|c|c|c|c|}
\hline & $\begin{array}{c}\text { Size }^{a} \\
(\mathrm{~nm})\end{array}$ & $\begin{array}{c}\text { PDI } \\
(\mathrm{nm})\end{array}$ & $\begin{array}{c}\text { Zeta } \\
\text { Potential } \\
(\mathrm{mV})\end{array}$ & $\begin{array}{c}\text { FA loading }^{\mathrm{c}} \\
(\mu \mathrm{F} \text { FA/ mg } \\
\text { NP })\end{array}$ & $\begin{array}{c}\text { EE }^{\mathrm{d}} \\
(\%)\end{array}$ \\
\hline NP-Z & $164 \pm 2$ & $0.07 \pm 0.01$ & $-46.0 \pm 1.5$ & - & - \\
\hline FA-NP-Z & $193 \pm 3$ & $0.20 \pm 0.06$ & $-29.3 \pm 3.1$ & $54 \pm 7$ & $57 \pm 6$ \\
\hline
\end{tabular}

${ }^{a}$ Determination of the nanoparticle size $(\mathrm{nm})$ by photon correlation spectroscopy

${ }^{\mathrm{b}}$ Determination of the zeta potential $(\mathrm{mV})$ by electrophoretic laser Doppler anemometry

${ }^{c}$ Amount of folic acid loaded in the nanoparticles ( $\mu \mathrm{g} \mathrm{FA} / \mathrm{mg}$ nanoparticles)

${ }^{d}$ Encapsulation efficiency (\%) 
Table 2. Pharmacokinetic parameters of folic acid administered as single dose of $1 \mathrm{mg} / \mathrm{kg}$ by the intravenous or oral routes as aqueous solution or loaded in zein nanoparticles. Data are expressed as mean \pm S.D, $(n=6)$.

\begin{tabular}{|c|c|c|c|c|c|c|c|c|c|}
\hline & Route & $\begin{array}{c}C_{\max } \\
(\mu \mathrm{g} / \mathrm{mL})\end{array}$ & $\begin{array}{c}T_{\max } \\
\text { (h) }\end{array}$ & $\begin{array}{c}\text { AUC } \\
(\mu g \mathrm{~h} / \mathrm{mL})\end{array}$ & $\begin{array}{l}\text { T1/2 } \\
\text { (h) }\end{array}$ & $\begin{array}{c}\mathrm{Cl} \\
(\mathrm{L} / \mathrm{h})\end{array}$ & $\begin{array}{l}\text { Vd } \\
\text { (L) }\end{array}$ & $\begin{array}{c}\text { MRT } \\
\text { (h) }\end{array}$ & $\begin{array}{l}\text { Fr } \\
(\%)\end{array}$ \\
\hline PBS & oral & - & - & - & - & - & - & - & - \\
\hline FA iv & iv & $5.5 \pm 2.7$ & 0.0 & $3.7 \pm 0.4$ & $1.2 \pm 0.6$ & $0.06 \pm 0.01$ & $0.10 \pm 0.05$ & $0.9 \pm 0.2^{* *}$ & 100 \\
\hline FA sol & oral & $0.2 \pm 0.0$ & $1.0 \pm 0.6$ & $1.3 \pm 0.3$ & $5.9 \pm 1.9$ & $0.06 \pm 0.02$ & $0.44 \pm 0.07$ & $5.7 \pm 1.6$ & 35 \\
\hline FA-NP-Z & oral & $0.4 \pm 0.1$ & $1.0 \pm 0.0$ & $3.0 \pm 1.0^{*}$ & $7.1 \pm 2.6$ & $0.05 \pm 0.01$ & $0.49 \pm 0.11$ & $6.5 \pm 1.3$ & 70 \\
\hline
\end{tabular}

$\mathrm{C}_{\text {max }}$ : peak plasma concentration; $\mathrm{T}_{\text {max }}$ : time to reach plasma concentration; $\mathrm{AUC}$ : area under the curve; $\mathrm{t} 1 / 2$ : half-life of the terminal phase; $\mathrm{Cl}$ : clearance; Vd: volume of distribution; MRT: mean residence time Fr: relative oral bioavailability

* Significant differences $(p<0.05)$ vs FA sol (Mann-Whitney-U)

**Significant differences $(p<0.01)$ vs FA sol (Mann-Whitney-U) 








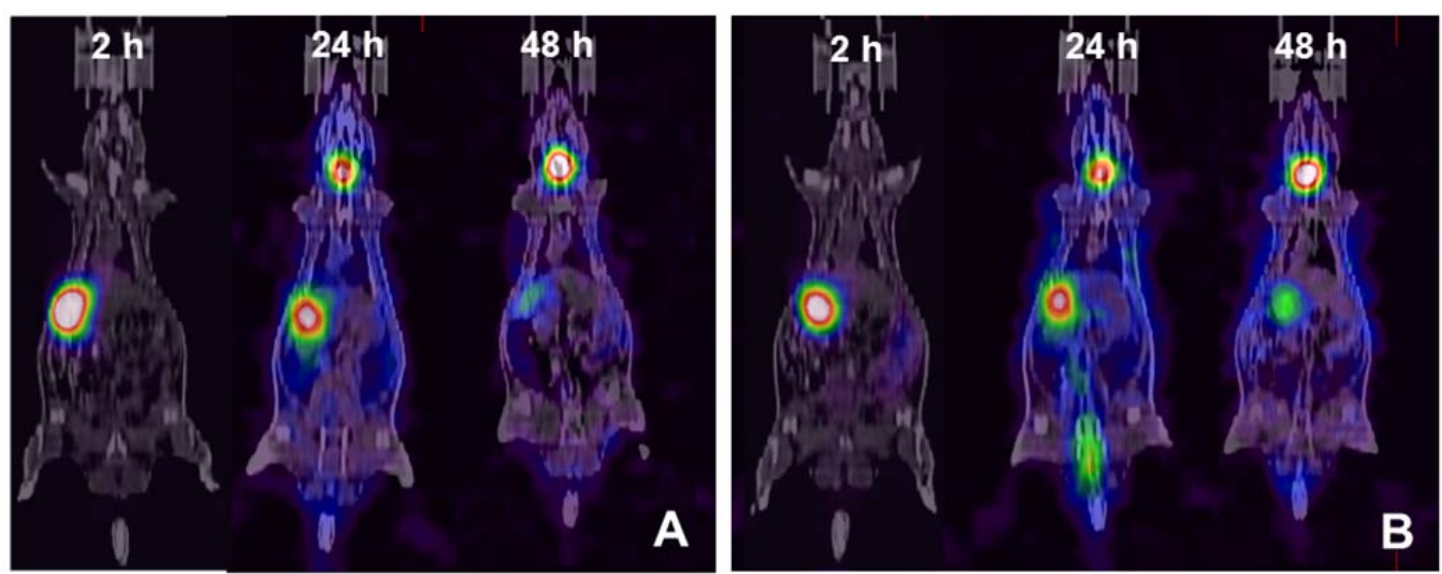


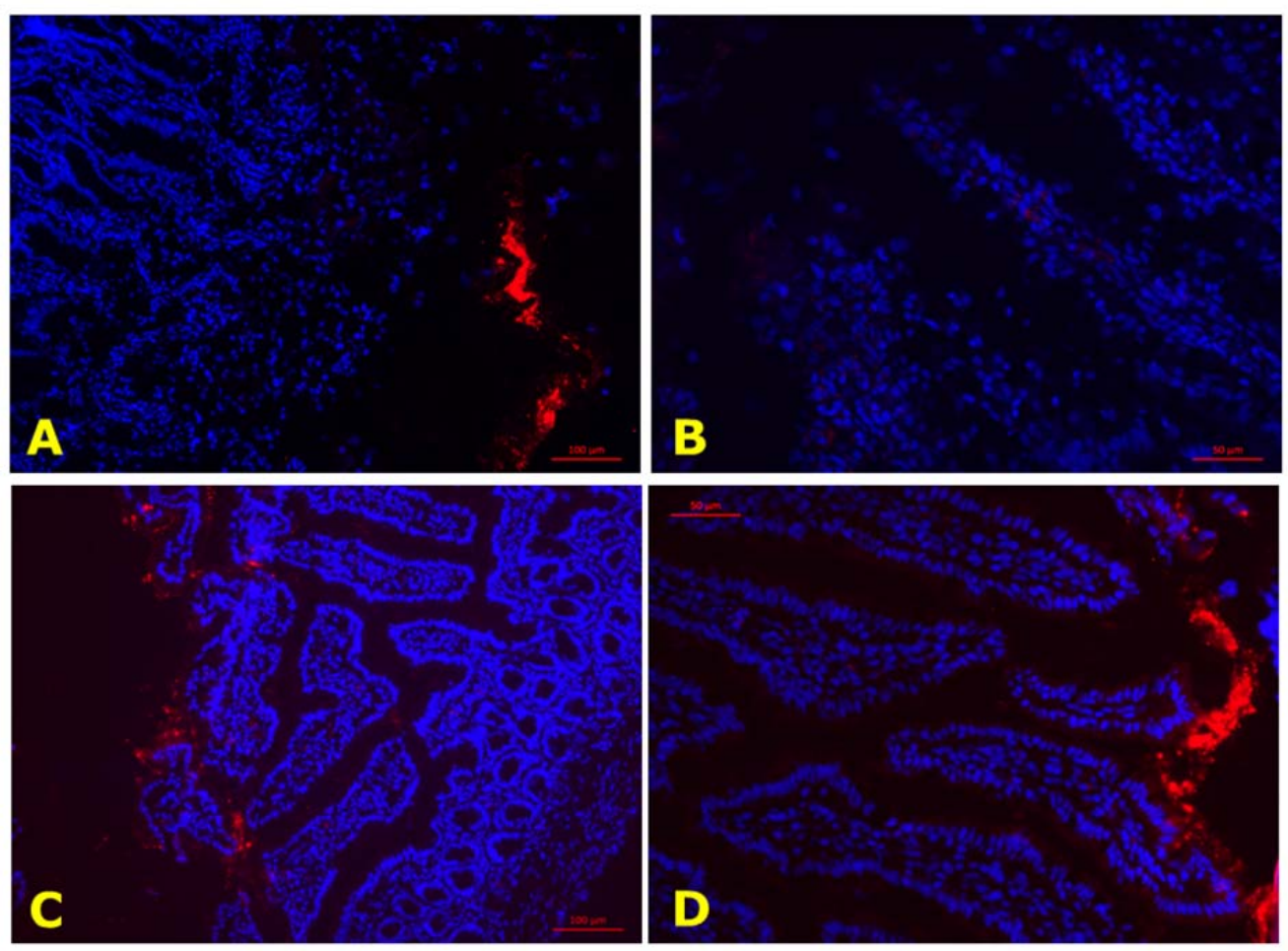




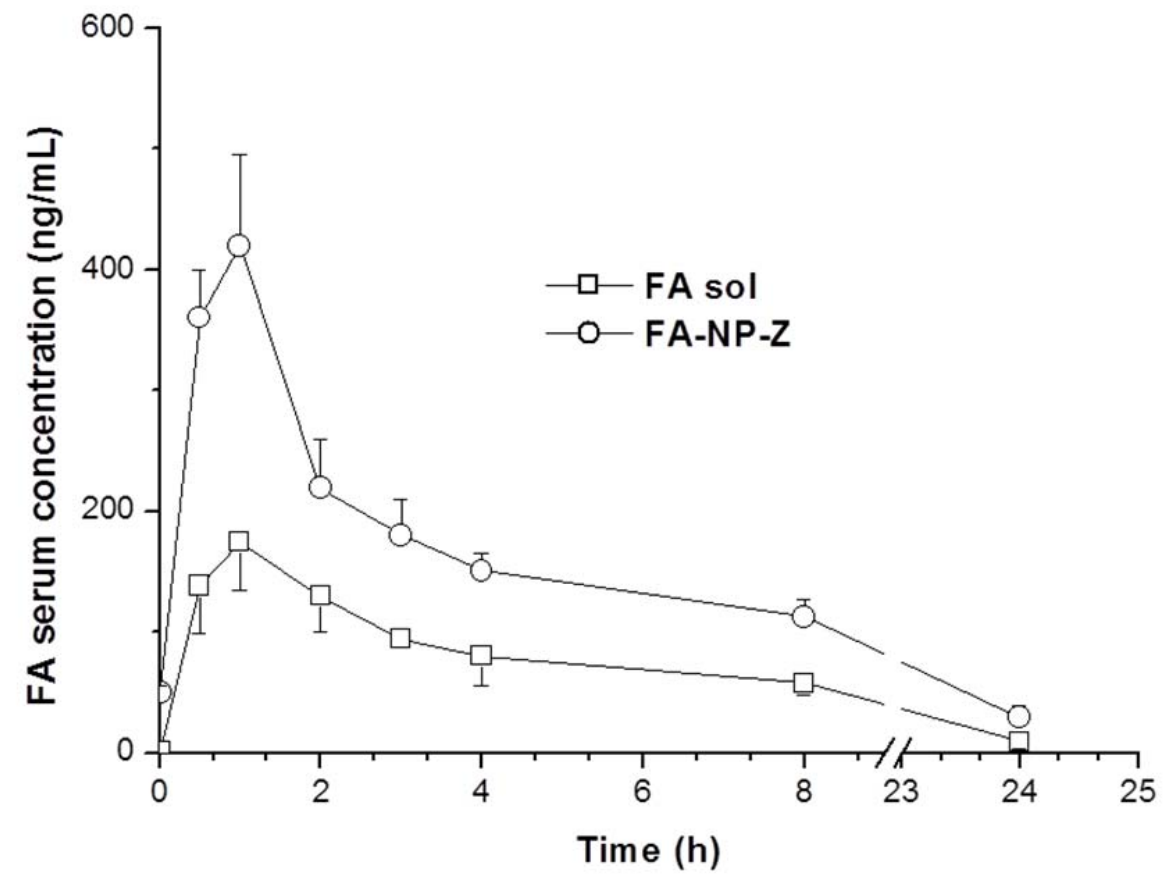




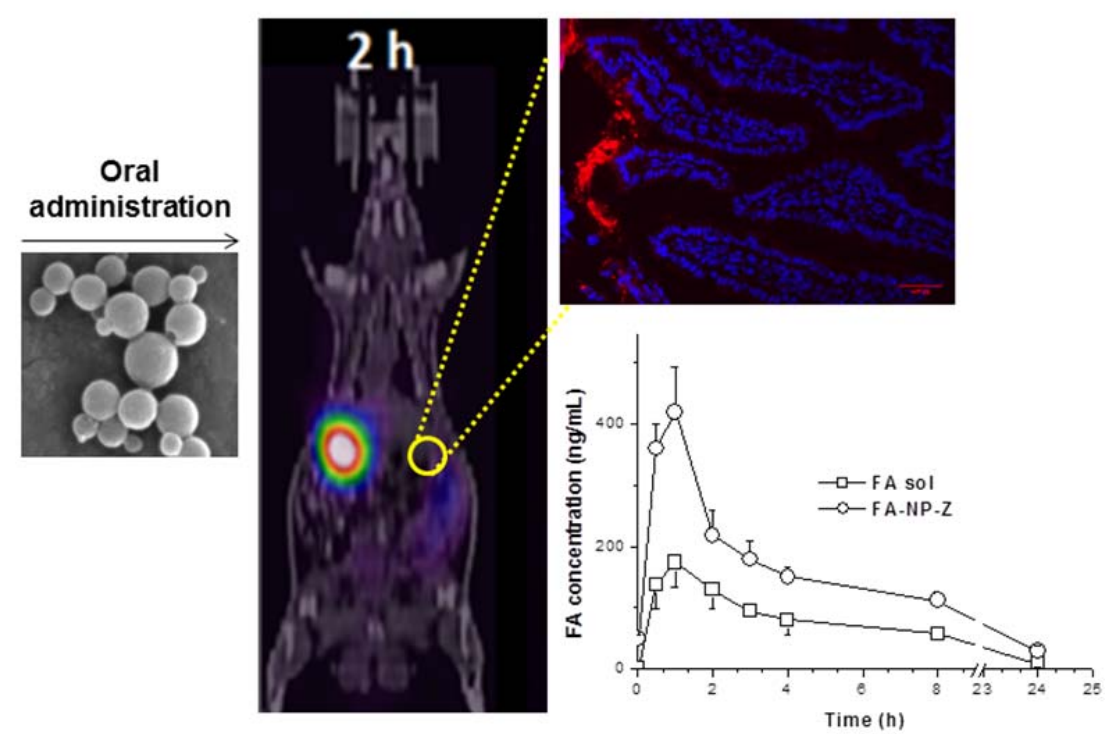






BY NC ND Esta obra está bajo una licencia de Creative Commons Reconocimiento-

NoComercial-SinObraDerivada 4.0 Internacional. 